\title{
Análisis del uso de micro convertidores DC/DC enfocados en la extracción máxima de energía en una granja fotovoltaica
}

\section{(Analysis of the use of micro DC/DC converters focused on the maximum extraction of energy in photovoltaic farm)}

\author{
Juan Vargas, ${ }^{1}$ Jorge Medina, ${ }^{1}$ Marcelo Pozo, ${ }^{1}$ Eduardo Ávila, ${ }^{1}$ Nataly Pozo, ${ }^{1}$ y Gabriel Salazar ${ }^{1}$
}

Resumen

Este trabajo presenta un estudio amplio del trabajo publicado en la revista INCISCOS 2018 títulado "Improving of the Photovoltaic High Power Plant Generation Using DC/DC Micro Converters";está enfocado en incrementar la cantidad de energía eléctrica generada de una Planta Fotovoltaica ubicada en Salinas, provincia de Imbabura-Ecuador, mediante la inclusión de micro convertidores DC/DC en los arreglos de los paneles fotovoltaicos y a partir de la implementación de una adecuada técnica de seguimiento del punto de máxima potencia. Los resultados indican que la inserción de los convertidores DC/DC en el sistema provocan un aumento en la cantidad de energía activa. Se realiza el análisis tanto con variaciones de la radiación solar como cambios en la temperatura ambiente.

\section{Palabras clave}

Control de generación de energía; paneles fotovoltaicos; celdas fotovoltaicas; rastreadores del punto de máxima potencia; conmutación de convertidores; convertidores DC/DC, gestión de energía.

\begin{abstract}
This work presents an extended study of the work published in the magazine INCISCOS 2018 titled "Improving of the Photovoltaic High Power Plant Generation Using DC/DC Micro Converters", and is focused on increase the amount of electrical energy generation of a Photovoltaic Plant located in Salinas, Imbabura Province (Ecuador) through the implementation of DC/DC converters in the solar panel arrays and tracking techniques of the maximum potency point. The results show that the insertion of the DC/DC converters in the system causes an increase in the amount of active power. The system is analyzed in variation of solar radiation as well as in the changes in the environment temperature.
\end{abstract}

\section{Keywords}

Power generation control; solar panels; photovoltaic cells; maximum power point trackers; switching converters; DC/ DC converters; energy management.

\section{Introducción}

Hoy en día, las centrales fotovoltaicas se consideran fuentes de generación de energía eléctrica amigables con el medio ambiente, ya que minimizan la emisión del dióxido de carbono a la atmósfera (Chen y Yan, 2015). La cantidad de energía eléctrica producida por una central solar dependerá, principalmente, de la radiación solar incidente y la temperatura ambiente (Benavides, Jurado y González, 2018). La mayoría de los sistemas fotovoltaicos basan su esquema de conexión en el tipo de instalación (ya sea modo isla o conectado a la red eléctrica) y a la cantidad de energía que manejan. En la Figura 1 se muestra la clasificación de las topologías empleadas en aplicaciones fotovoltaicas (Blaabjerg, Orlowska-Kowalska y Rodríguez, 2014). Para el presente caso se emplea un inversor centralizado debido a la gran cantidad de energía que debe manejar el sistema.

1 Escuela Politécnica Nacional, Quito, Ecuador (\{juan.vargas01, jorge.medinaj, marcelo.pozo, nataly.pozo, gabriel.salazar\} @ epn.edu.ec; pato_ab1990@hotmail.com). 
Figura 1. Topología de conexiones de sistemas fotovoltaicos

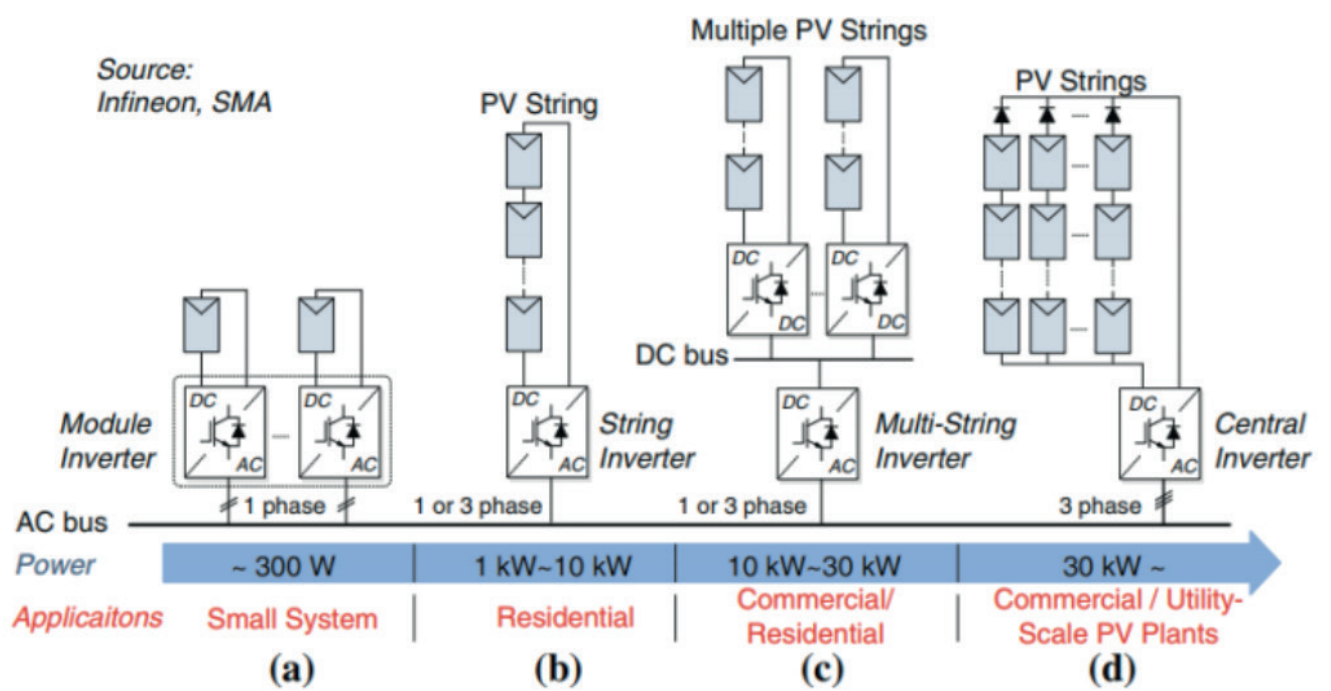

(Blaabjerg, Orlowska-Kowalska y Rodríguez, 2014).

Otro aspecto importante a considerar para incrementar la eficiencia energética de una central de generación eléctrica basada en paneles fotovoltaicos es la selección de un adecuado algoritmo de búsqueda de punto de máxima potencia (Maximum Power Point Tracking (MPPT, por su sigla en inglés,). Los algoritmos MPPT basan su funcionamiento en el análisis de la curva Potencia vs. Voltaje (Figura 2) en donde se rastrea el punto de máxima potencia-MPP y se obliga al sistema a operar en dicho punto (Ávila, Pozo, Pozo y Salazar, 2018).

Figura 2. Curva potencia vs. voltaje de un arreglo fotovoltaico

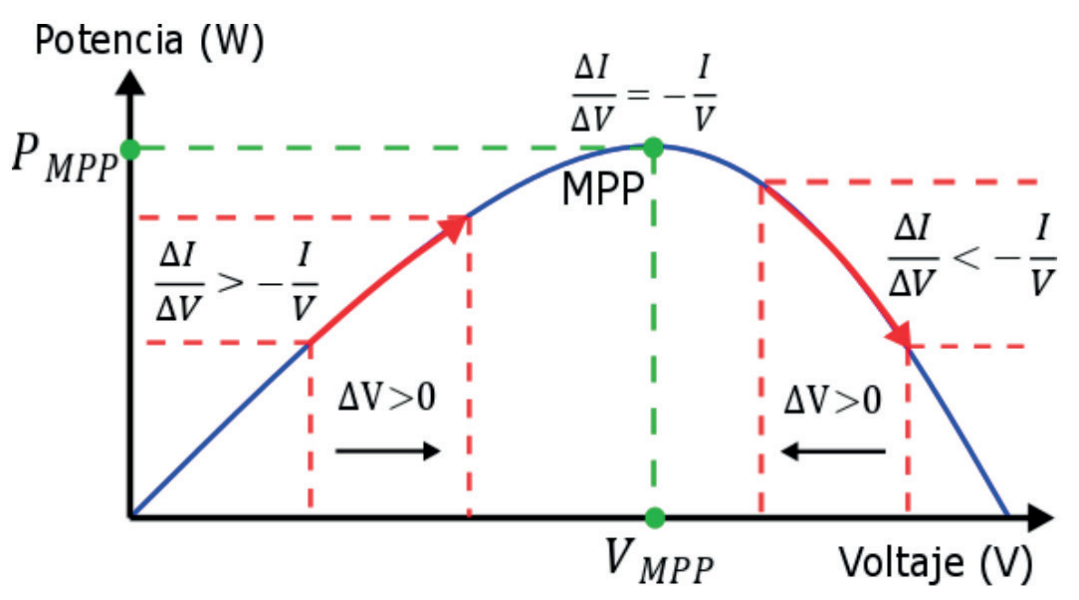

(Ávila, 2017)

Cabe destacar que en la mayoría de los estudios, se analiza bajo radiación uniforme incidente sobre los arreglos de paneles solares. Sin embargo, el fenómeno conocido como Sombras Parciales Partial Shading Conditions (PSC, por su sigla en inglés,) reduce significativamente la 
eficiencia del sistema y crea los denominados puntos locales de máxima potencia Local Maximum Power Point (LMPP, por sus siglas en inglés)), tal como se muestra en la Figura 3 (Ji, Jung, Kim, Kim, Lee y Won, 2011). Por este motivo, es imprescindible analizar las prestaciones de los diferentes algoritmos MPPT (Tabla 1) haciendo un énfasis particular en su desempeño durante condiciones de sombras parciales.

Figura 3. Curvas características de los paneles fotovoltaicos ante la presencia de sombras parciales

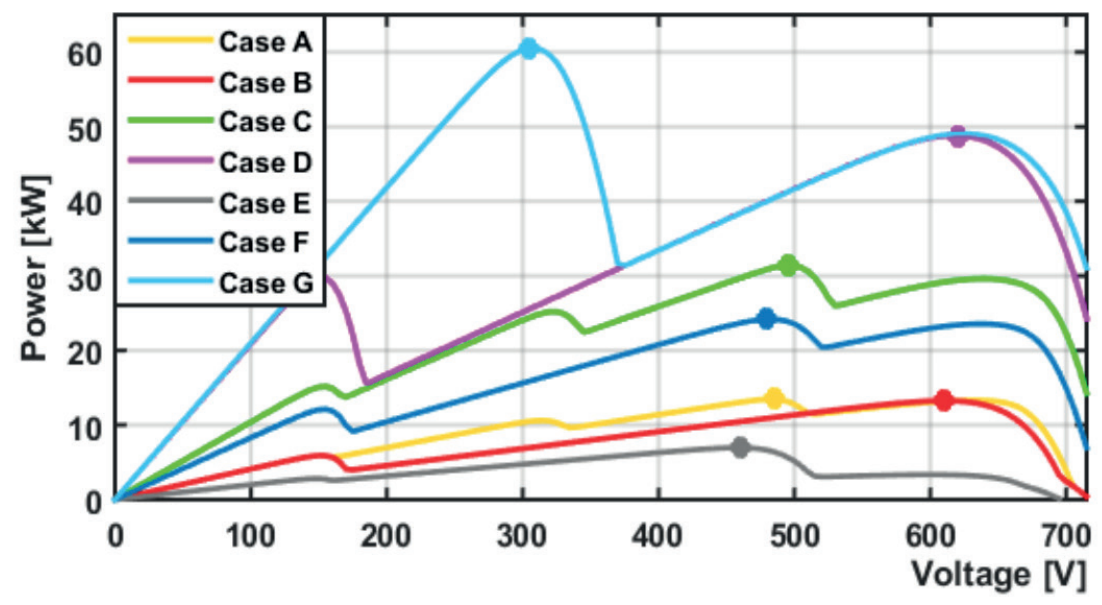

Tabla 1. Parámetros comparativos de los algoritmos MPPT (Cruz de Lima, 2012)

\begin{tabular}{|l|l|l|l|l|l|l|}
\hline \multicolumn{1}{|c|}{ Algoritmo MPPT } & \multicolumn{1}{c|}{ P\&O } & \multicolumn{1}{c|}{ Cl } & \multicolumn{1}{c|}{ LD } & \multicolumn{1}{c|}{ HC } & \multicolumn{1}{c|}{ RN } & \multicolumn{1}{c|}{ PSO } \\
\hline Dependencia del panel fotovoltaico & No & No & Si & No & Si & No \\
\hline Parámetros medidos & V,I & V,I & Varia & V,I & Varia & V, I \\
\hline Velocidad de convergencia & Variable & Variable & Rápida & Variable & Rápida & Rápida \\
\hline Complejidad de implementación & Baja & Media & Alta & Baja & Alta & Alta \\
\hline Detección de LMPP & No & No & Si & No & Si & Si \\
\hline
\end{tabular}

\section{Metodología}

La central fotovoltaica considerada en este estudio genera aproximadamente 3MW de potencia activa. Cabe destacar que esta central se encuentra dividida en dos etapas de similar configuración, pero de diferente potencia nominal ( 1 y $2 \mathrm{MW}$, respectivamente). En este estudio se analiza para la etapa de 1MW. En la Figura 4 se puede observar el diagrama unifilar que describe las etapas del sistema de generación de energía eléctrica.

Mediante la introducción de los convertidores DC/DC en los arreglos de los paneles fotovoltaicos y la aplicación de técnicas de control, se busca extraer la mayor cantidad de energía del sistema. Para esto, se analiza el desempeño del sistema empleando tres diferentes topologías DC/DC de convertidores estáticos de potencia: buck-boost, cuk y sepic (Figura 5). 
Figura 4. Diagrama unifilar del Sistema fotovoltaico

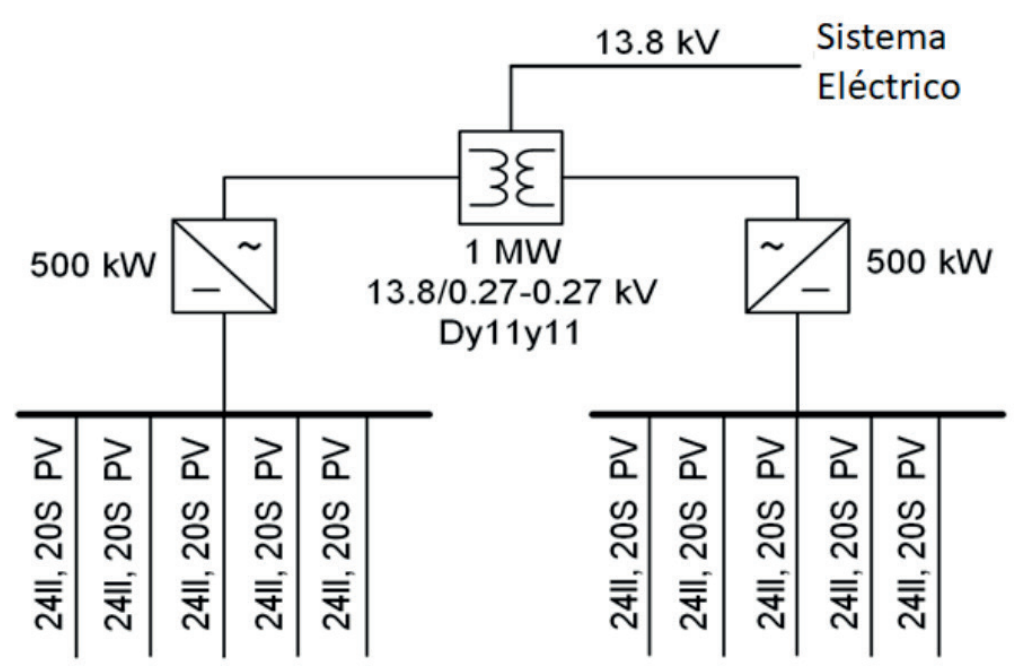

Figura 5. Topologías de los conversores DC/DC

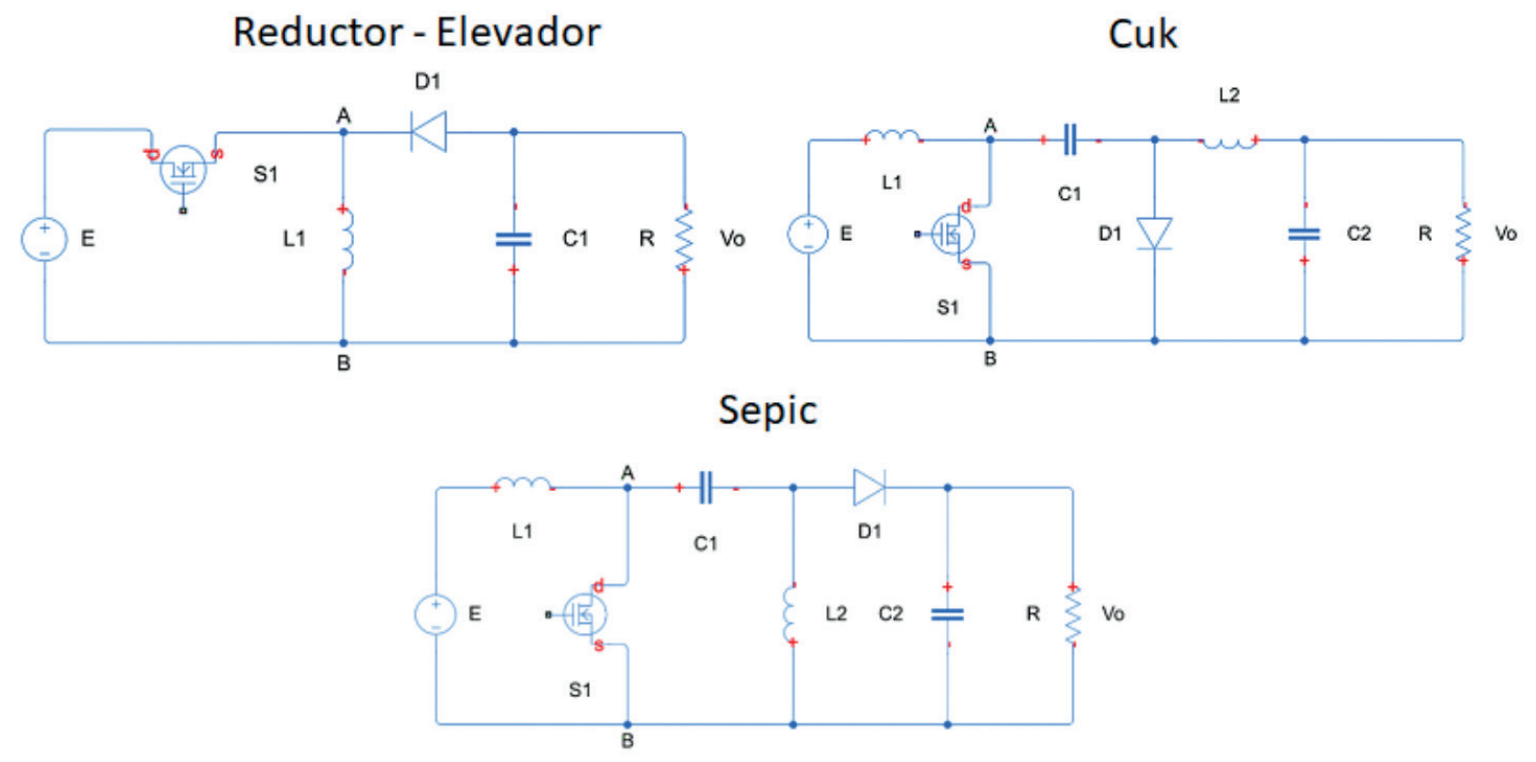

En cuanto al esquema de control empleado, se realizó la conmutación del semiconductor de potencia de las tres topologías de los convertidores DC/DC, mediante el esquema de control que se muestra en la Figura 6, en donde se hace uso de un algoritmo MPPT encargado de generar el voltaje de referencia al cual se espera que trabajen los arreglos de paneles solares. Adicionalmente, en el esquema de control se emplea un control en cascada en el cual el lazo interno (SMC) es encargado de controlar la corriente circulante a la entrada de cada convertidor y en el lazo externo se realiza el control de voltaje mediante un controlador PI (Camacho, Domínguez, Leica y Rosales, 2016). 
Figura 6. Esquema de control para la conmutación de los convertidores DC/DC

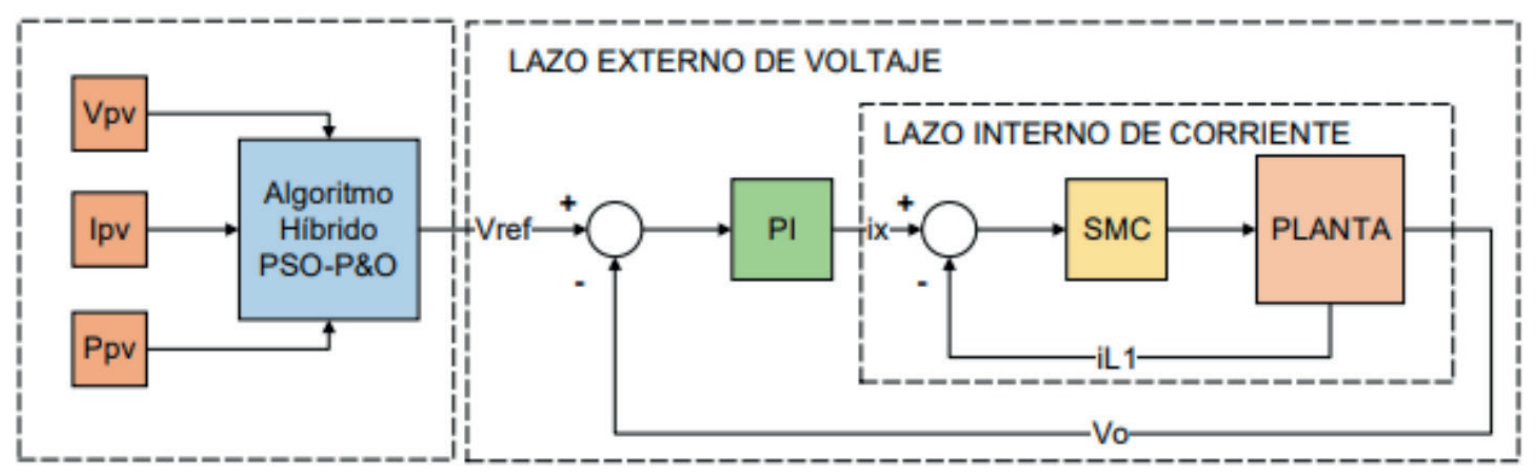

(Vargas, 2018)

En el desarrollo del algoritmo MPPT se propone un método basado en la rápida convergencia del algoritmo de P\&O y la capacidad para garantizar una operación en el MPP global característica del algoritmo de PSO (Figura 7). Es así que en la fase de exploración el algoritmo de PSO dispone las partículas sobre la curva P-V y determina cuál de estas partículas genera la mayor producción energética (G_best). Mientras que en la fase de convergencia se propone emplear el algoritmo de P\&O para desplazar a la partícula G_best a través de la curva P-V del arreglo fotovoltaico y alcanzar el MPP global. Es decir, en la fase de convergencia, en lugar de trabajar con un enjambre de partículas, se empleará únicamente la partícula G_best. Cabe destacar que este cambio reduce la complejidad y el número de iteraciones del algoritmo, factores que permiten incrementar la eficiencia del sistema (Ávila, 2017) (Vargas, Medina, Pozo, Pozo y Salazar, 2018).

Figura 7. Fases del algoritmo PSO-P\&O

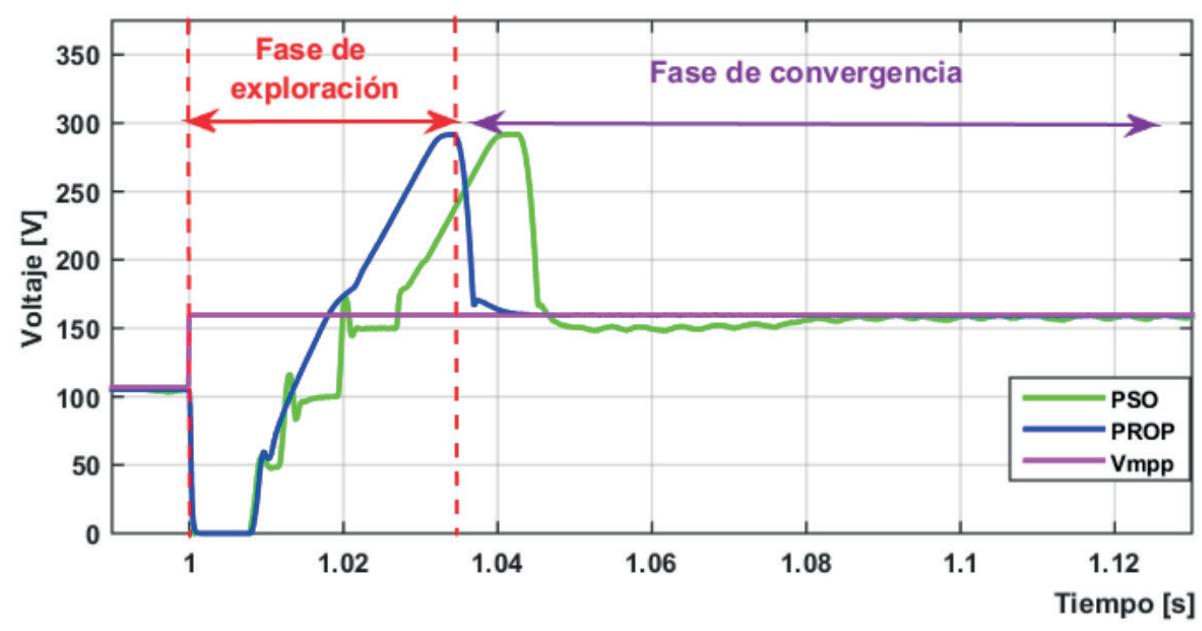

(Ávila, 2017).

Por otra parte, se considera como entradas el voltaje y corriente que generan los arreglos de los paneles solares y como salida se origina un voltaje de referencia que a su vez representa el punto de máxima potencia de los paneles solares. En este caso el algoritmo PSO-P\&O está 
conformado por dos etapas. La primera etapa es la encargada de rastrear el punto de máxima potencia, mediante el uso de enjambre de partículas. Para este caso de estudio se toma como referencia 70 partículas en las que se considera que se estará moviendo el punto de máxima potencia, tomando en cuenta las curvas características de Potencia-Voltaje de los paneles solares. Una vez conocido el punto de máxima potencia la segunda etapa del algoritmo se encarga de realizar la convergencia del voltaje generado por lo paneles solares hacia el punto de máxima potencia. Alcanzado el punto de máxima potencia entra en funcionamiento el algoritmo P\&O encargado de mantener al sistema oscilando alrededor del punto de máxima potencia.

La ley del controlador en modo deslizante encargado de controlar la corriente circulante a la entrada de cada convertidor se representa en las siguientes ecuaciones, para el convertidor buck-boost (1), cuk (2) y sepic (3).

$$
\begin{gathered}
U(t)=\frac{V_{o}}{V_{o}+E}+K_{D} \frac{S(t)}{|S(t)|+\rho} \\
U(t)=1-\frac{E}{v_{c 1}}+K_{D} \frac{S(t)}{|S(t)|+\rho} \\
U(t)=1-\frac{E}{v_{c 1}+V_{o}}+K_{D} \frac{S(t)}{|S(t)|+\rho}
\end{gathered}
$$

Donde:

$V:$ Voltaje de salida del convertidor.

E: Voltaje de entrada del convertidor.

$S(t)$ : Superficie deslizante.

$V_{c i}:$ Voltaje en los terminales del capacitor.

$K_{D}:$ Parámetro responsable del acercamiento.

$\rho$ : Parámetro responsable de las oscilaciones.

El criterio de estabilidad se encuentra descrito por tres condiciones, que de cumplirse estas, se puede concluir que el sistema es estable (López, 2014).
1. $V(t)>0$
2. $\mathrm{V}(0)=0$
3. $\dot{\mathrm{V}}(\mathrm{t})<0$

Es necesario escoger una candidata de Lyapunov $\vee$ que cumpla con los dos primeros criterios de estabilidad, en este caso es descrita por la siguiente ecuación:

$$
V=\frac{1}{2} S^{2}>0
$$

Por último, es necesario aplicar el tercer criterio de estabilidad:

$$
V \dot{(t)}=S(t) \dot{S(t)}<0
$$


De esta manera, como resultado del análisis de estabilidad se tiene las siguientes ecuaciones, para el convertidor buck-boost (6), cuk (7) y sepic (8).

$$
\begin{gathered}
S(t)\left[-\frac{E+V_{o}}{L 1} K_{D} \frac{S(t)}{|S(t)|+\rho}\right]<0 \\
S(t)\left[-\frac{v_{C 1}}{L 1} K_{D} \frac{S(t)}{|S(t)|+\rho}\right]<0 \\
S(t)\left[-\frac{v_{C 1}+V_{o}}{L 1} K_{D} \frac{S(t)}{|S(t)|+\rho}\right]<0
\end{gathered}
$$

Mientras se cumpla que, $E_{,} V_{C 1^{\prime}} V_{0^{\prime}} L_{1} K_{D} y$ tomen valores positivos. Permite concluir que la candidata de Lyapunov para los tres convertidores DC/DC es estable.

El desarrollo del controlador proporcional-integral del lazo externo de voltaje se basa en el método de la curva de reacción, resultando que los valores de las constates del controlador después de realizar una sintonización se muestran en la Tabla 2.

Tabla 2. Parámetros del controlador PI

\begin{tabular}{|l|c|c|}
\hline \multicolumn{1}{|c|}{ DC/DC Converter } & Kp & Ki \\
\hline Buck-Boost & 0.7 & 150 \\
\hline Cûk & 0.2 & 150 \\
\hline Sepic & 0.2 & 150 \\
\hline
\end{tabular}

\section{Resultados}

Los resultados que se presentaran a continuación, se los obtuvo de la comparación del modelo con y sin convertidores DC/DC en los arreglos de paneles solares, realizando dos pruebas en donde se varia la temperatura y la radiación solar incidente en los paneles solares. Además, se considera dos ubicaciones para insertar los convertidores DC/DC, siendo a la salida de cada caja de conexión (CC, por su sigla en inglés) y a la salida de cada caja de conexiones de strings (CCS por sus siglas en inglés). En la Figura 8 se muestra una subdivisión de los arreglos de los paneles solares con la finalidad de poder simular la presencia de sombras parciales (Vargas, Medina, Pozo, Pozo y Salazar, 2018).

Figura 8. División del área de los arreglos de paneles solares

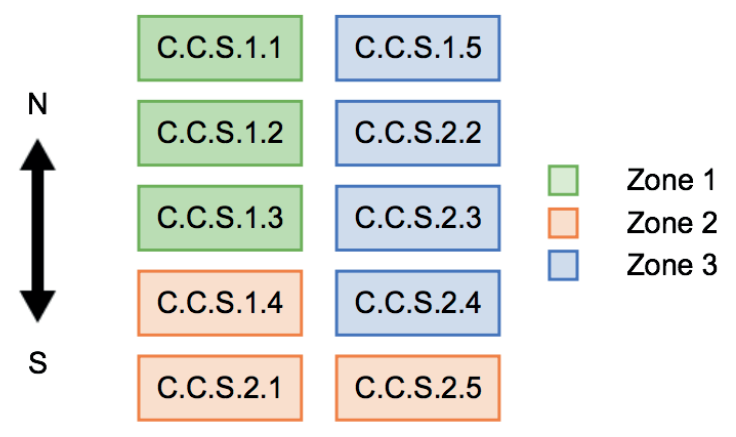


La primera prueba a la que sometió el sistema desarrollado presenta variaciones en los perfiles radiación, mientras que los perfiles de temperatura se mantienen constantes como se muestra en la Figura 9.

De esta manera, bajo el perfil de radiación variable se presentan los resultados en las Figura 10 y Figura 11, al comparar el modelo con y sin convertidores DC/DC, considerando las dos ubicaciones en donde van a ser insertados. Como se puede evidenciar el desempeño de los conversores cuk y sepic son similares y en este caso se presenta un incremento mayor al insertar los convertidores a la salida de cada C.C.S.

Figura 9. Perfiles de radiación solar y de temperatura ambiente
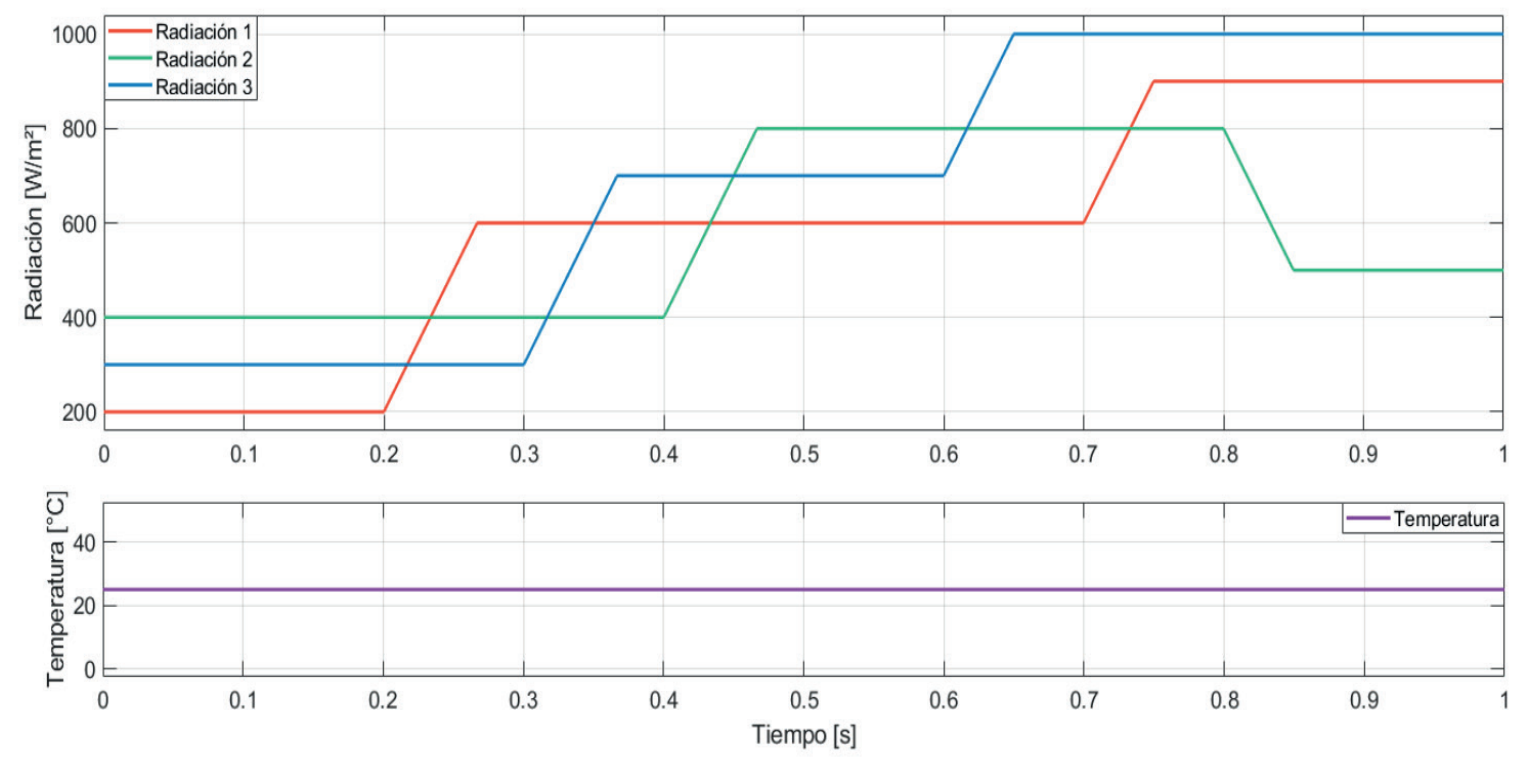

Figura 10. Potencia activa generada por el modelo con y sin convertidores DC-DC

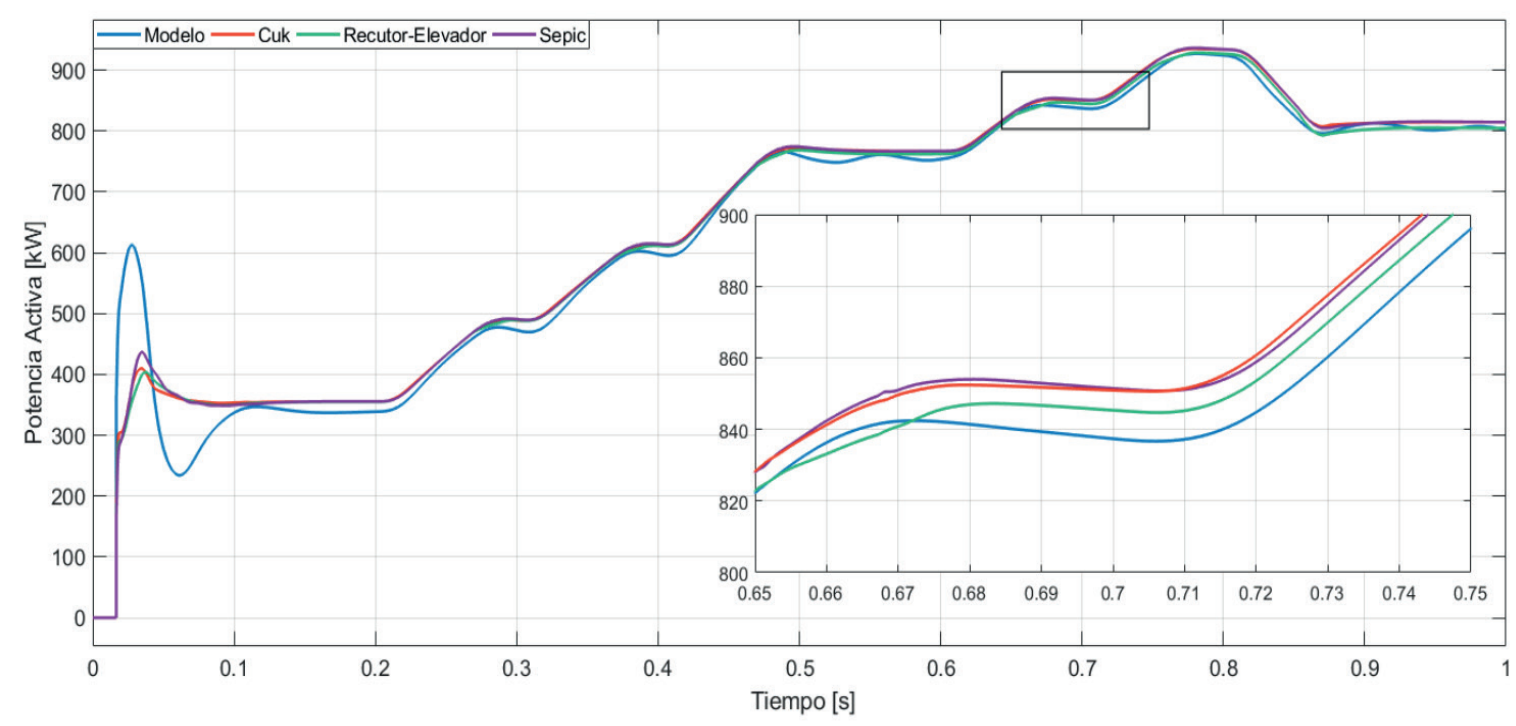


Figura 11. Potencia activa generada por el modelo con y sin convertidores DC-DC

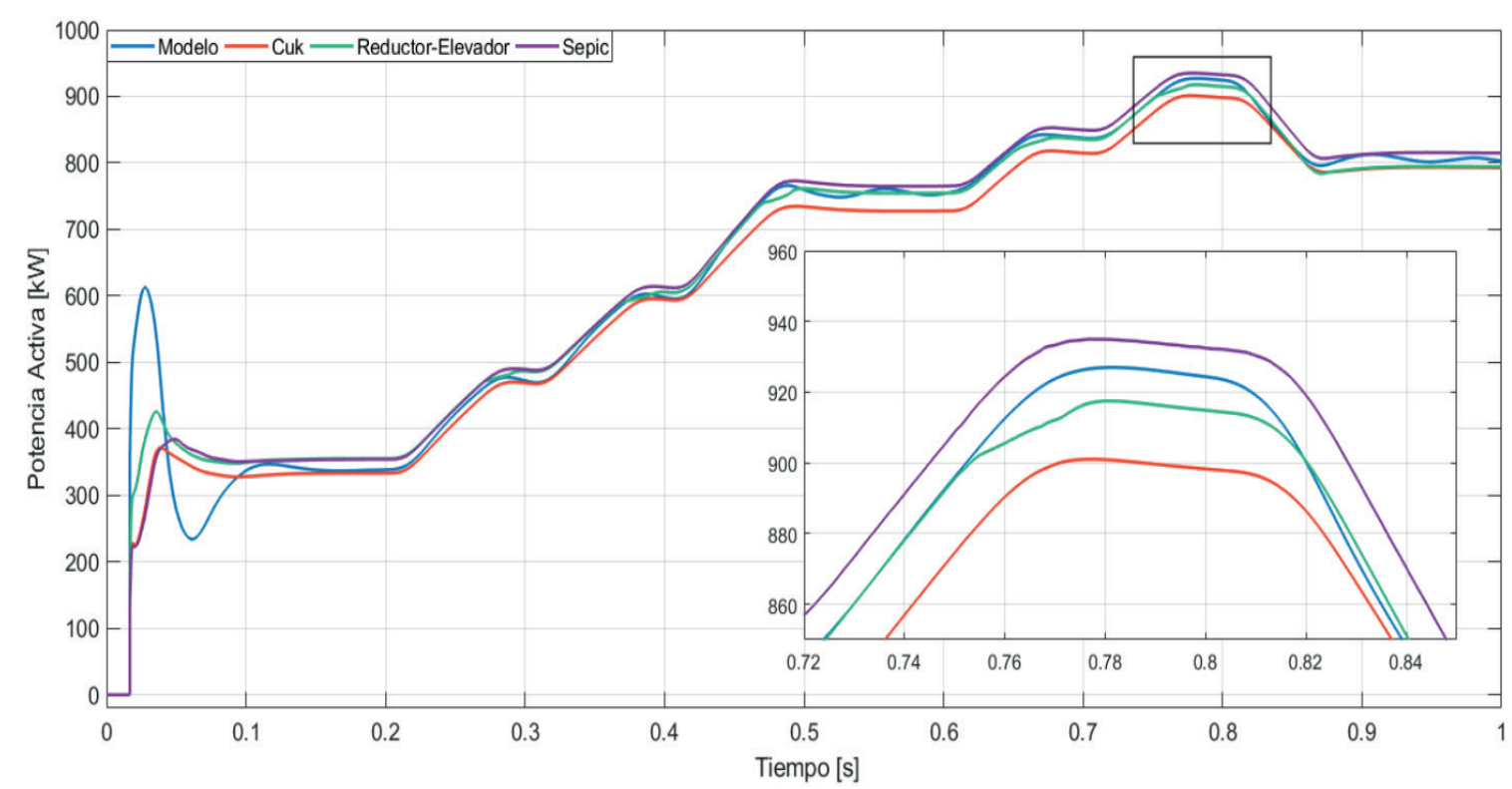

En el diagrama de barras de la Figura 12. se puede observar un incremento en la cantidad de energía 2.99 kWh, cuando se inserta convertidores sepic a la salida de cada C.C.S.

Figura 12. Diagrama de barras de la potencia activa generada

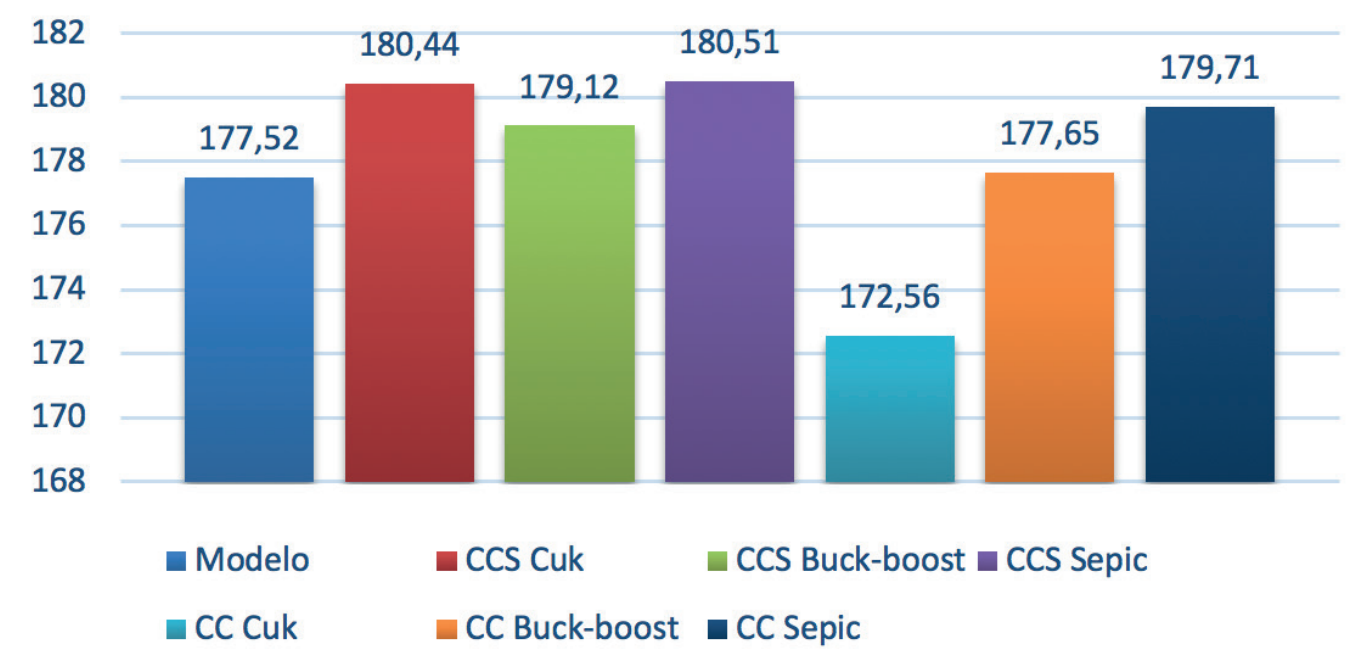

Por otra parte, en la Figura 13. se puede observar el funcionamiento del algoritmo PSOP\&O, el cual es aplicado en el convertidor DC/DC para una radiación solar variable, el punto de máxima potencia tiende a ser el mismo ya que la corriente generada por los arreglos de los paneles solares no variará. 
Figura 13. Funcionamiento del algoritmo PSO-P\&O

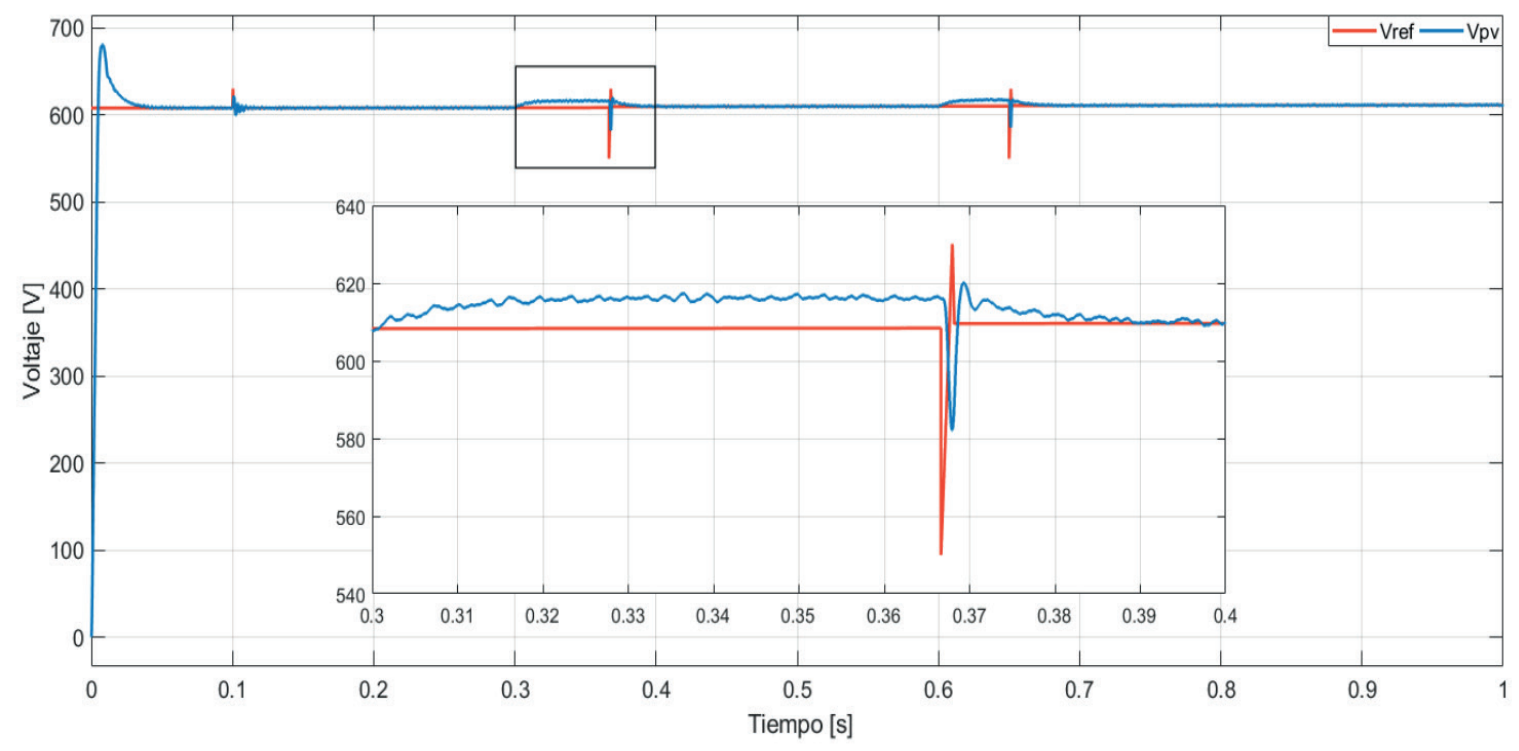

La segunda prueba a la que fue sometido el sistema desarrollado, mantiene los perfiles de radiación constante y los perfiles de temperatura son variables como se muestra en la Figura 14.

Figura 14. Perfiles de radiación solar y temperatura ambiente
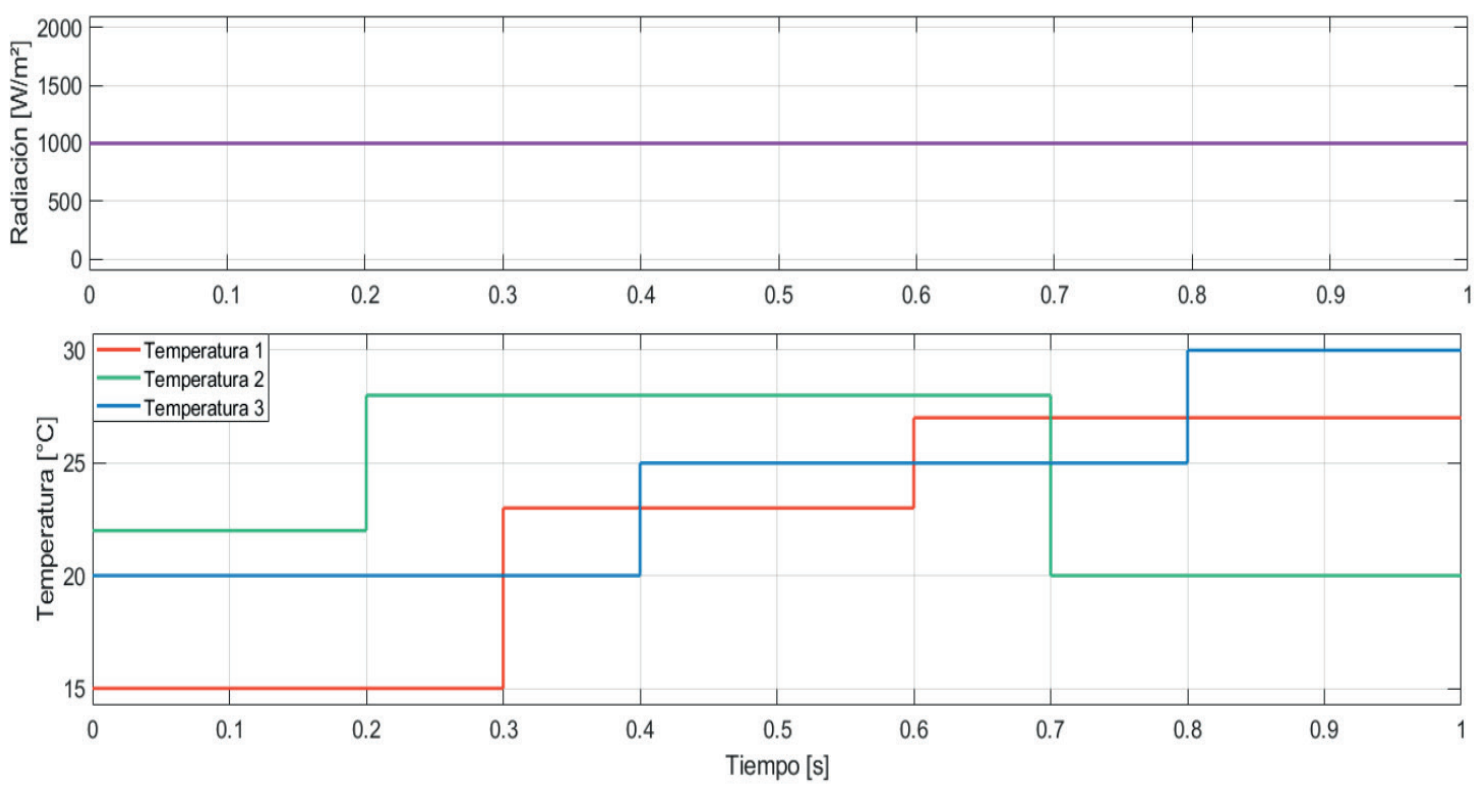

Los resultados obtenidos del modelo con y sin convertidores DC/DC se muestra en la Figura 15 y a la salida de cada C.C. se muestra en la Figura 16, evidenciando un incremento en la cantidad de energía generada al trabajar con los convertidores cuk y sepic. 
Figura 15. Potencia activa generada por el modelo con y sin convertidores DC-DC a la salida de cada C.C.S

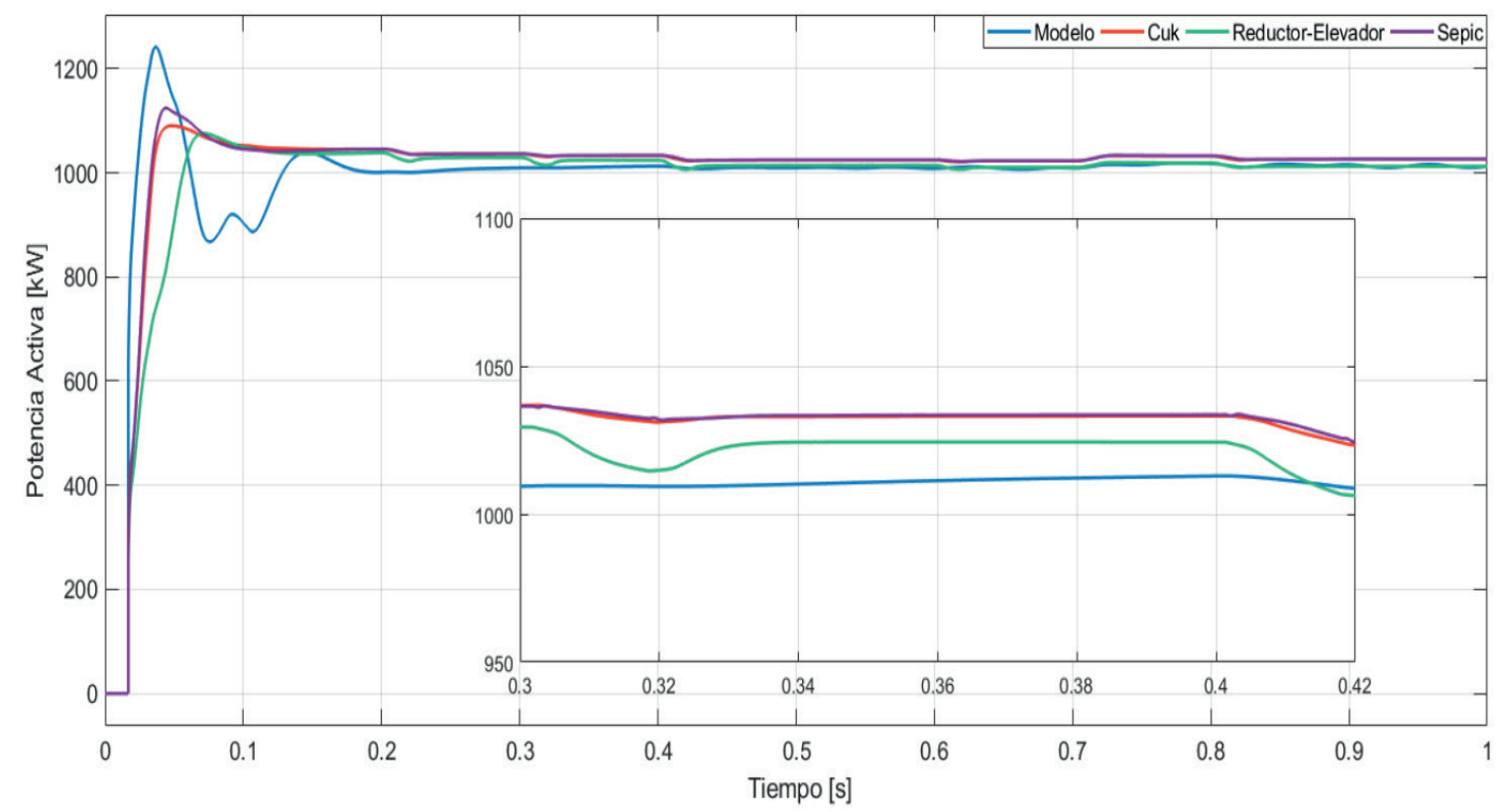

Mientras en la Figura 17 se detalla la cantidad de energía obtenida en cada caso al insertar los convertidores $\mathrm{DC} / \mathrm{DC}$, de esta manera se tiene un incremento de $5.03 \mathrm{kWh}$ cuando se insertan los convertidores sepic a la salida de cada C.C.S.

En la Figura 18 se puede apreciar el funcionamiento del algoritmo PSO-P\&O cuando se trabaja con una variación en el perfil de temperatura, en donde el punto de máxima potencia presenta ciertos escalones debido a la variación de temperatura.

Figura 16. Potencia activa generada por el modelo con y sin convertidores DC-DC a la salida de cada C.C

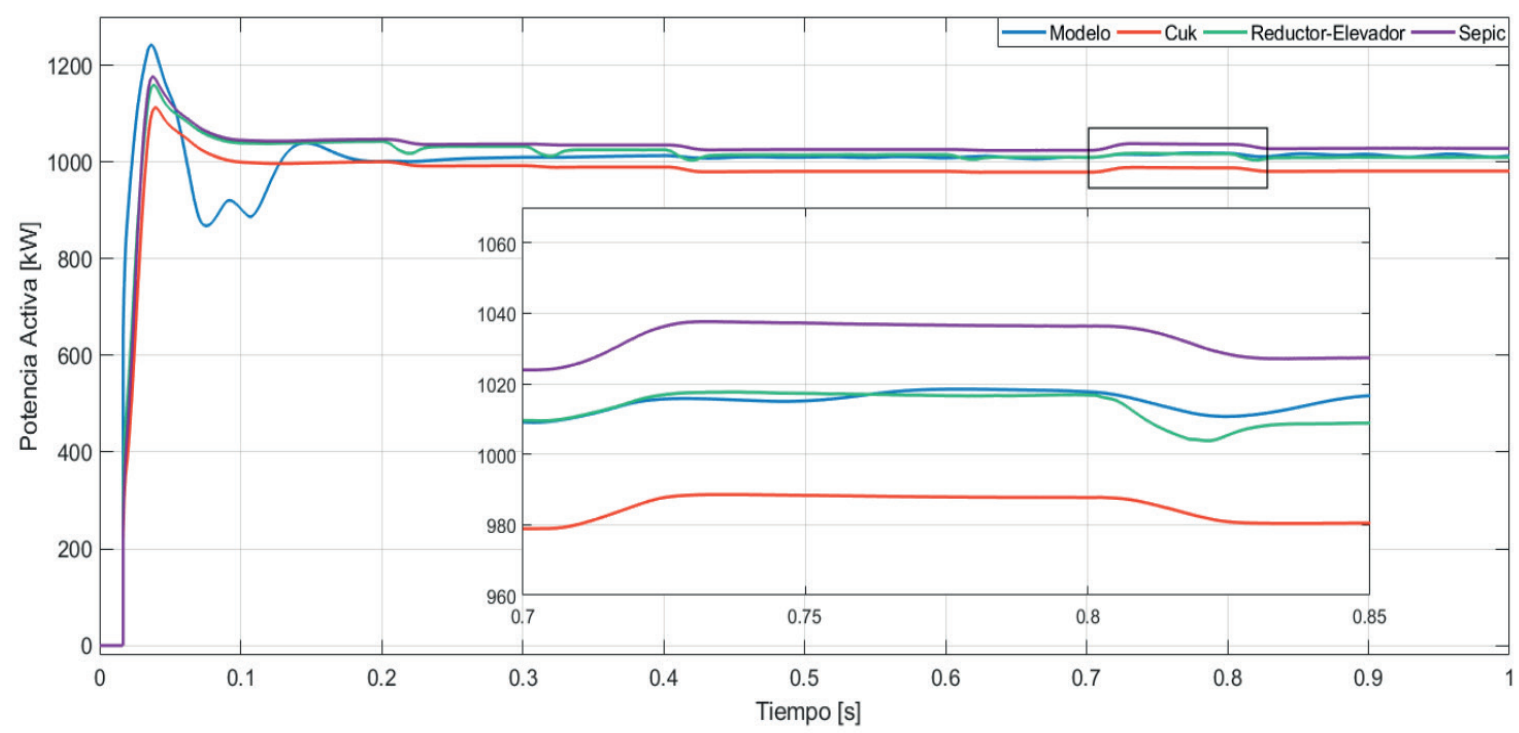


Figura 17. Diagrama de barras de la potencia activa generada

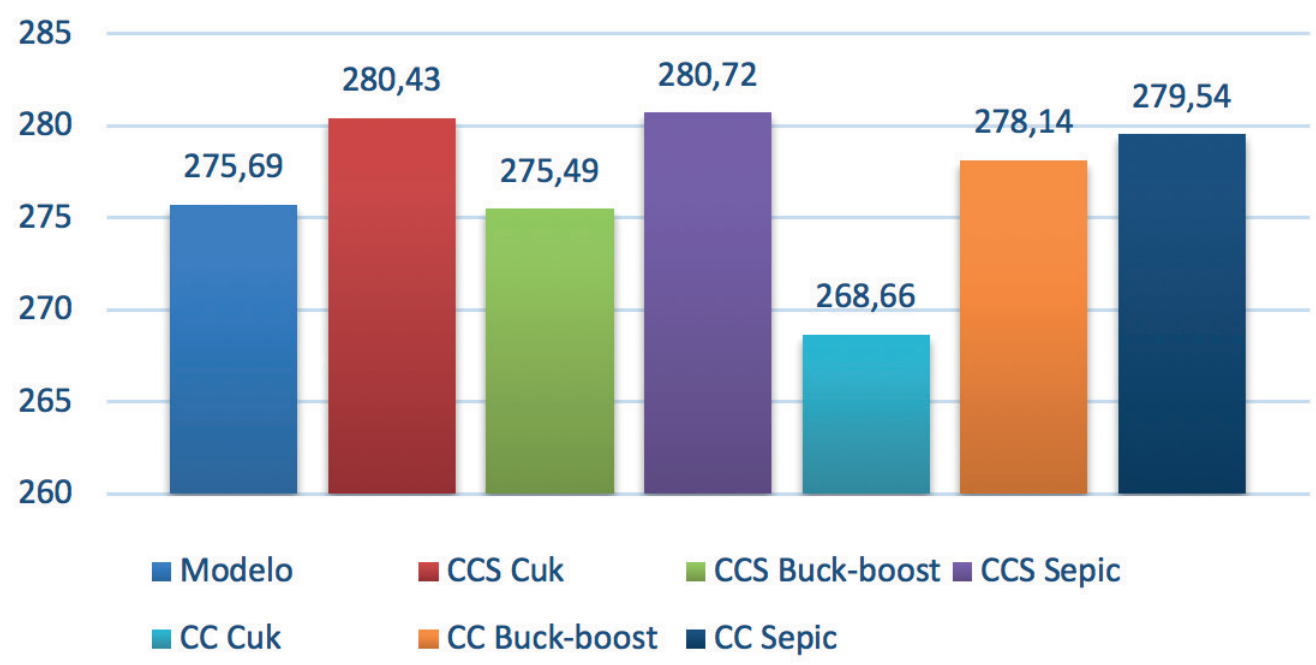

Figura 18. Funcionamiento del algoritmo PSO-P\&O

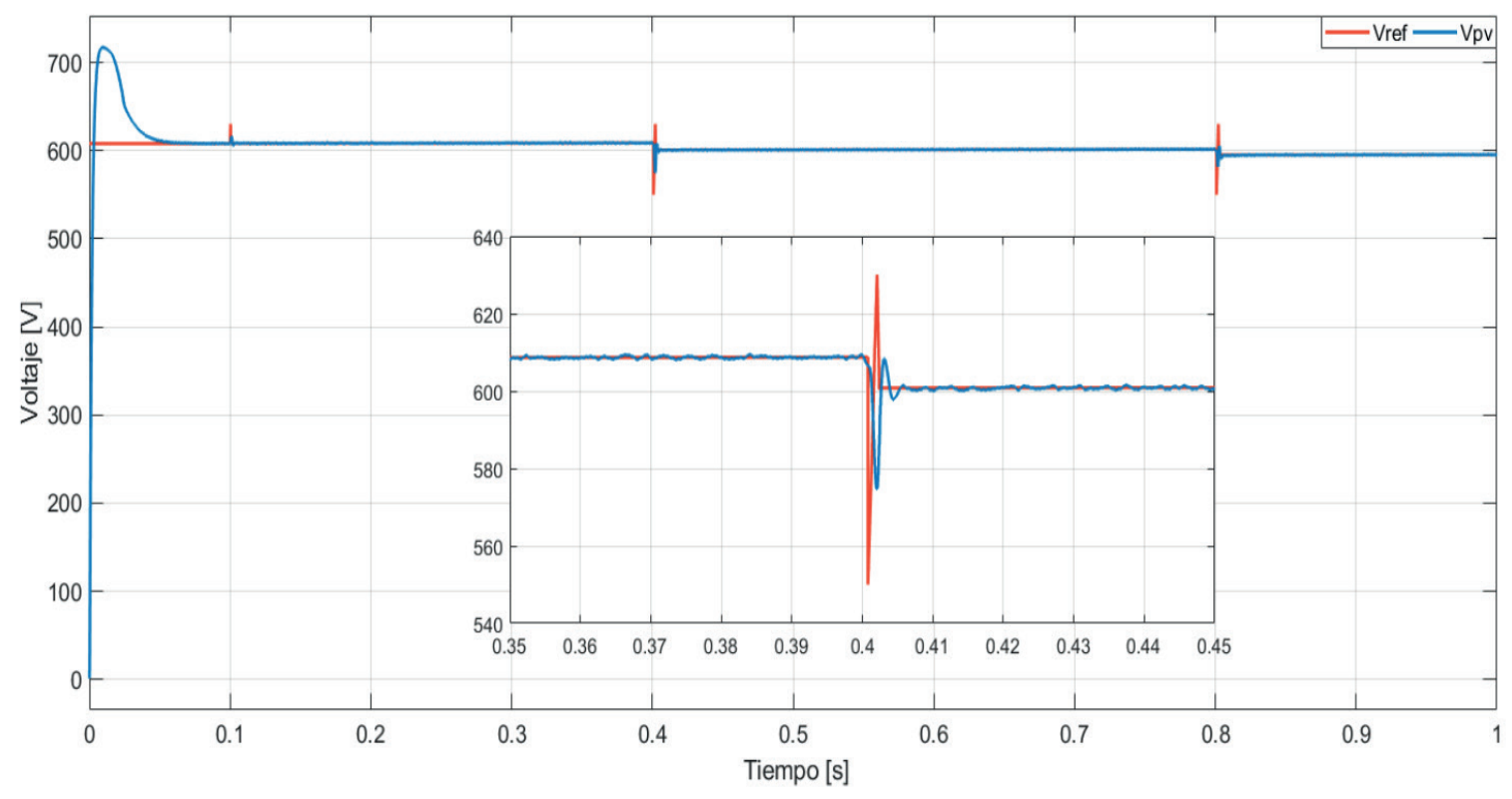

\section{Conclusiones y recomendaciones}

A partir de los resultados del trabajo de la inserción de los convertidores DC / DC, aplicando el algoritmo PSO-P\&O en la etapa de generación de energía eléctrica de la planta de energía fotovoltaica, la cantidad de energía generada por la planta de energía solar podría incrementarse en un $0.01 \%$ cada día.

En términos de ubicación ideal donde se pueden insertar convertidores DC / DC, es más factible colocarlos en la salida de cada C.C.S. de los arreglos de paneles solares. 
Si bien, los tres convertidores cuk, sepic y buck-boost se adaptan para trabajar en sistemas fotovoltaicos, los mejores resultados obtenidos en términos de la cantidad de energía generada son los convertidores cuk y sepic.

En cuanto a las pruebas, se realizan variaciones en la radiación y la temperatura para verificar el correcto funcionamiento del algoritmo MPPT implementado, porque cuando solo varía la radiación, la tensión generada por los paneles solares tiende a permanecer constante y los valores correspondientes a la corriente varían dependiendo de la cantidad de radiación incidente en ese momento, mientras que si la temperatura varía solo, los valores de voltaje dependerán del valor de radiación aplicado y la corriente permanecerá constante.

\section{Referencias}

Ávila, E. (2017). Diseño y simulación de un control en cascada (PI-Deslizante) para los convertidores presentes en una planta virtual de generación de energía eléctrica-. Quito: Escuela Politécnica Nacional.

Ávila, E., Pozo, N., Pozo, M. y Salazar, G. (2018). Improved Particle Swarm Optimization Based MPPT for PV Systems under Partial Shading Conditions. Quito: Southern Power Electronics Conference (SPEC).

Benavides, D., Jurado, F. y González, L., (2018). Date analysis and tools applied to modeling and simulation of PV system in Ecuador. ENFOQUE UTE, 1-12.

Blaabjerg, F., Orlowska-Kowalska, T. y Rodríguez, J. (2014). Advanced and Intelligent Control in Power Electronics and Drivers. Nueva York: Springer.

Chen, P., y Yan, B. (2015). A Comparative Study on MPPT for Photovoltaic Generation Systems. IEEExplore, 1-6.

Cruz de Lima, N. (2012). Micro inversor para módulo fotovoltaico. Porto: Universidad do Porto.

Domínguez, X., Camacho, O., Leica, P., y Rosales, A. (2016). A Fixed-Frequency Sliding-mode Control in a Cascade Scheme for the Half-bridge Bidirectional DC-DC Converter". IEEExplore.

Ji, Y.-H, Jung, D.-Y, Kim, J.-G, Kim, J.-H., Lee, T.-W. y Won, C.-Y. (2011). A Real Maximum Power Point Tracking Method for Mismatching Compensation in PV Array Under PArtially Shaded Conditions. IEEE TRANSACTIONS ON POWER ELECTRONICS, 1001-1009.

Liu, C.L, Liu, Y.-H, Luo, Y.-F, y Huang, J. W (2012). "A PSO-based MPPT Algorithm for Photovoltaic Systems Subject to Inhomogeneous Insolation". IEEExplore, 721-726.

López, F. (2014). Construcción de funciones de Lyapunov para Sistemas Homogéneos de Segundo Orden (Método por reducción de variable). CDMX, México: Universidad Nacional Autónoma de México.

Vargas, J., Medina, J., Pozo, M., Pozo, N. y Salazar, G. (2018). Improving of the Photovoltaic High Power Plant Generation Using DC/DC Micro Converters. Quito: IEEE.

Vargas, J. (2018). "Diseño y Simulación de la Optimización en la Generación de Energía en una Central Fotovoltaica Mediante Conversores DC/DC y la Técnica de Control MPPT", Quito: Escuela Politécnica Nacional. 